\title{
ECG Denoising Methodology using Intrinsic Time Scale Decomposition and Adaptive Switching Mean Filter
}

\author{
B.T. Krishna, P.S. Kameswari
}

\begin{abstract}
Electrocardiogram (ECG) is a widely employed tool for the analysis of cardiac disorders. A clean ECG is often desired for proper treatment of cardiac ailments. However, in the real scenario, ECG signals are corrupted with various noises during acquisition and transmission. In this article, an efficient ECG de-noising methodology using combined intrinsic time scale decomposition (ITD) and adaptive switching mean filter (ASMF) is proposed. The standard performance metric namely output SNR improvement measure the efficacy of the proposed technique at various signal to noise ratio (SNR). The proposed de-noising methodology is compared with other existing ECG de-noising approaches. A detail qualitative and quantitative study and analysis indicate that the proposed technique can be used as an effective tool for de-noising of ECG signals and hence can serve for better diagnostic in computer-based automated medical system. The performance of the proposed work is compared with existing ECG de-noising techniques namely wavelet soft thresholding based filter (DWT) [16], EMD with DWT technique [18], DWT with ADTF technique [19]. The effectiveness of the presented work has been evaluated in both qualitative and quantitative analysis. All the simulations are carried out using MATLAB software environment.
\end{abstract}

Keywords: Adaptive Switching Mean Filter, Electrocardiogram, Intrinsic Time Scale Decomposition, Signal to Noise Ratio.

\section{INTRODUCTION}

Electrocardiogram (ECG) is commonly utilized for the identification of the cardiovascular diseases. ECG reflects the electrical activities of the cardiac system.[1-3]. Due to the rapid growth of population and lack of proper infrastructure, computer based automated ECG analyzer has become a vital tool for early diagnosis of the cardiac diseases as it does a fast processing also [4]. However, for precise feature extraction of ECG signal, good quality noise free signals are typically desired. In the real scenario, various noises like Gaussian

Manuscript received on 01 March 2021 | Revised Manuscript received on 06 March 2021 | Manuscript Accepted on 15 May 2021 | Manuscript published on 30 May 2021.

* Correspondence Author

Dr Battula Tirumala Krishna*, Professor, Department of Electronics and Communication Engineering JNTUK Kakinada, India. Tkbattula@gmail.com

Putti Siva Kameswaari, M.Tech, Deaprtment Electronics and Communication Engineering, JNTUK Kakinada, India. siva9putti@gmail.com

(C) The Authors. Published by Lattice Science Publication (LSP). This is an open access article under the CC-BY-NC-ND license (http://creativecommons.org/licenses/by-nc-nd/4.0/) noise, muscle artifacts, power line interference, and baseline wander noise contaminate the ECG signal during its acquisition and transmission [5]. Elimination of these noises is an essential task for the proper diagnosis of the cardiac diseases from the signal features and hence it is an active field of research[6].

Various researchers have contributed numerous methods to address the development of computer-based automated ECG denoising. These developed techniques are mainly based on finite impulse response (FIR) filter $[7,8]$, adaptive filter [5], neural net-work [9], principle component analysis (PCA) [10], independent component analysis (ICA) [11], non-local mean (NLM) filter [12], extended Kalman filter (EKF) [13], discrete wavelet transform(DWT) filtering [14-16], empirical mode decomposition (EMD)[17], EMD with DWT [18], adaptive dual threshold filtering [19].FIR based filtering techniques $[7,8]$ can only remove the noise components those are outside the frequency range of ECG signal. Moreover, this type of methods do not preserve the low-frequency ECG components ( $\mathrm{P}$ and $\mathrm{T}$ waves). Adaptive filter and neural net-work based systems [5,9] require additional reference signals and training phase, hence not suitable for real-time applications.

In PCA and ICA techniques [10,11], the derived statistical model is much sensitive to a small change in the signals or the noises. Moreover, for ICA based approaches, visual inspection of the independent components are crucial which is not feasible for long-term applications. Extended Kalman Filter [13] based technique involves manual initialization of parameters, which are associated with amplitude, width, and phase of each component of a complete ECG cycle. Discrete Wavelet Transform [14-16] assisted soft and hard thresholding based de-noising is popular for filtering of non-stationary signals. Another method based on EMD is quite effective for dealing with the ECG signals[20]. In [17,18], a window is applied to preserve the QRS complexes in the low order IMFs that leaves the noise components in the QRS regions. The adaptive dual threshold filter (ADTF) based technique [19] rejects the initial sub-bands of the wavelet decomposed signal causing significant information loss in the higher frequency region. The Intrinsic time scale decomposition (ITD) method overcomes many of the limitations of both classical (e.g. Fourier transform or wavelet transform based) and more recent (empirical mode decomposition based) approaches to TFE analysis of signals that are nonlinear and/or non-stationary in nature. In this article, the effectiveness of ITD and adaptive switching mean filter (ASMF) are considered together for developing an efficient ECG de-noising technique.

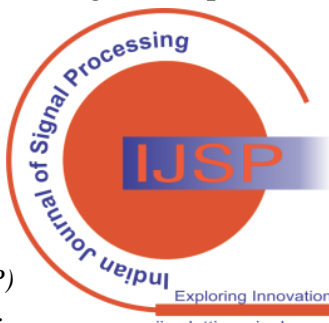


This approach effectively reduces the noise components in QRS regions and enhances the QRS complexes. Subsequently, an ASMF technique is utilized to decrease the effect of noises in the low-frequency region of the ECG signals and to improve the signal quality further. The Paper is organized as follows. In Section 2, Intrinsic Time Scale Decomposition of ECG signal is proposed. Results and Discussions are presented in Section 3. Finally Conclusions are drawn in Section 4.

\section{PROPOSED ECG DENOISING METHODOLOGY}

The outline of the ASMF based ECG denoising approach is demonstrated in Fig. 1. A detail discussion on every step is given below.

\section{A. Intrinsic Time Scale decomposition (ITD) of ECG}

In this paper, we use a method called the intrinsic time-scale decomposition (ITD).The ITD overcomes the limitations of EMD listed earlier, as well as those previously mentioned and associated with more classical approaches such as Fourier and wavelets. In particular, the ITD provides: (i) efficient signal decomposition into 'proper rotation' components for which instantaneous frequency and amplitude are well defined.

(ii) precise temporal information regarding instantaneous frequency and amplitude of component signals with a temporal resolution equal to the time-scale of occurrence of extrema in the input signal,

(iii) a new class of real-time signal filters that utilize the newly available instantaneous amplitude and frequency/phase information together with additional features and morphology information obtained via single-wave analysis.

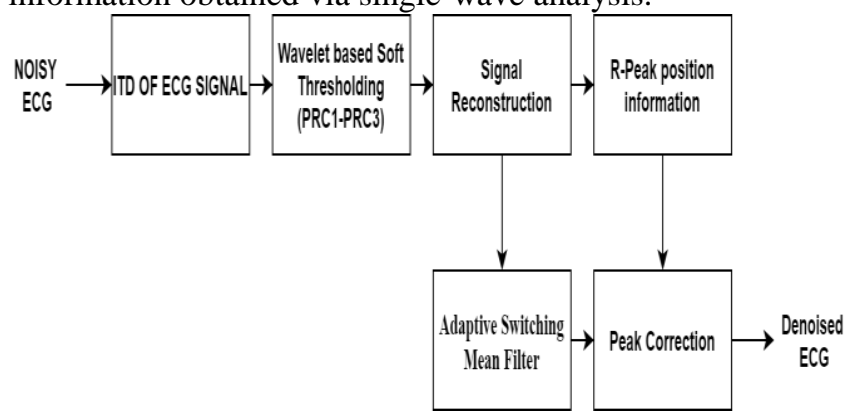

Figure. 1. Proposed ECG Denoising method outine Algorithm:

Given a signal $\mathrm{X}_{\mathrm{t}}$ we define an operator, $\mathrm{L}$, which extracts a baseline signal from $\mathrm{X}_{\mathrm{t}}$ in a manner that causes the residual to be a proper rotation. More specifically, $\mathrm{X}_{\mathrm{t}}$ can be decomposed as:

$$
X_{t}=L X_{t}+(1-L) X_{t}=L_{t}+H_{t}
$$

Where $\mathrm{L}_{\mathrm{t}}=\mathrm{LX}_{\mathrm{t}}$ the baseline is signal and $\mathrm{H}_{\mathrm{t}}=(1-\mathrm{L}) \mathrm{X}_{\mathrm{t}}$ is a proper rotation.Suppose $\left\{\mathrm{X}_{\mathrm{t}}, \mathrm{t}>0\right\}$ is a real-valued signal, and let $\left\{\tau_{k}, k=1,2,.\right\}$ denote the local extrema of $X_{t}$, and for convenience define $\tau_{0}=0$. In the case of intervals on which $\mathrm{X}_{\mathrm{t}}$ is constant, but which contain extrema due to neighboring signal fluctuations, $T_{k}$ is chosen as the right endpoint of the interval. To simplify notation, let $\mathrm{X}_{\mathrm{k}}$ and $\mathrm{L}_{\mathrm{k}}$ denote $\mathrm{X}\left(\tau_{\mathrm{k}}\right)$ and $\mathrm{L}\left(\tau_{\mathrm{k}}\right)$, respectively. Suppose that $\mathrm{L}_{\mathrm{t}}$ and $\mathrm{H}_{\mathrm{t}}$ have been defined on $\left[0, \tau_{\mathrm{k}}\right]$ and that $\mathrm{X}_{\mathrm{t}}$ is available for $t \in\left(0, \tau_{k+2}\right]$. We can then define a (piece-wise linear) baseline-extracting operator, $\mathrm{L}$, on the interval $\left(\tau_{k}, \tau_{k+1}\right]$ between successive extrema as follows:

$$
\begin{aligned}
& L X_{t}=L_{t}=L_{k}+\left(\frac{L_{k+1}-L_{k}}{X_{k+1}-X_{k}}\right)\left(X_{t}-X_{k}\right) t \in \\
& \left(\tau_{k}, \tau_{k+1}\right]
\end{aligned}
$$

Where

$$
\begin{aligned}
& L_{k+1}=\alpha\left[X_{k}+\left(\frac{\tau_{k+1}-\tau_{k}}{\tau_{k+2}-\tau_{k}}\right)\left(X_{k+2}-X_{k}\right)\right]+(1- \\
& \alpha) X_{k+1}
\end{aligned}
$$

and $0<\alpha<1$ is typically fixed with $\alpha=1 / 2$. We construct the baseline signal, $\mathrm{L}_{\mathrm{t}}$, in this manner in order to maintain the monotonicity of $\mathrm{X}_{\mathrm{t}}$ between extrema, while at the same time remaining inside an 'envelope' generated by some wave riding atop this baseline.

After defining the baseline signal according to equations (2) and (3), we are able to define the residual, proper-rotation-extracting operator, $\mathrm{H}$, as

$$
H X_{\mathrm{t}}=(1-L) X_{\mathrm{t}}=H_{\mathrm{t}}=X_{\mathrm{t}}-L_{\mathrm{t}}
$$

A typical noisy ECG signal and its PRCs are presented in Fig.2. Here, the noisy ECG signal is decomposed into eight PRCs (PRC1to PRC8) and a residue signal. From PRC1 to residue signal, a decreasing nature of oscillation is noticed. The lower order PRCs (PRC1-PRC3) contains high-frequency signal information (QRS complex) and noises are mainly spread over these PRCs. In conventional ITD based ECG de-noising method [20], to reduce the noises, these lower order PRCs are rejected.

\section{B. Wavelet based de-noising (PRC1-PRC3)}

The lower order PRCs (PRC1-PRC3) contain high-frequency artifacts as well as QRS complexes [18]. To remove the noises from these IMFs, a wavelet soft thresholding based de-noising technique is applied. In, this method, the signal is divided into a set of approximation and detail coefficients.
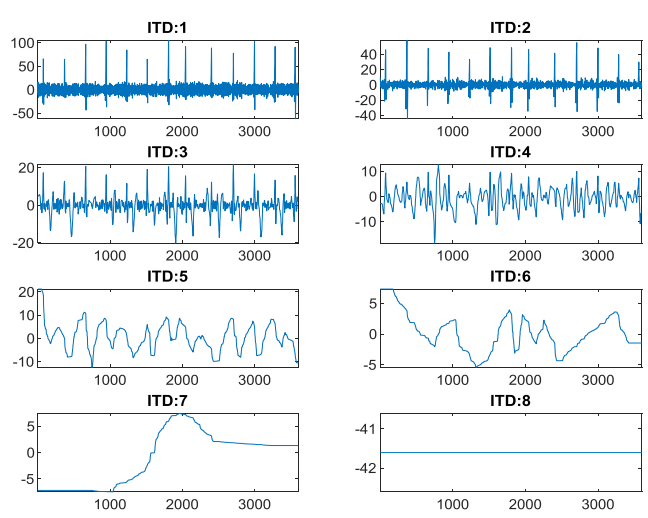

Figure. 2. Decomposed components using ITD technique

As detail coefficients contain the high-frequency information, these coefficients are threshold according to some predefined rule.

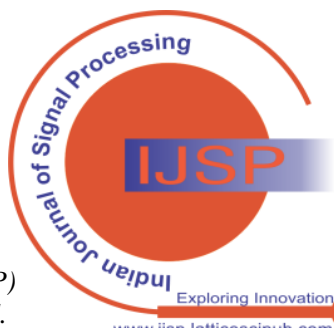


Then, by applying the inverse wavelet transform, the filtered signal is extracted from the updated coefficients. The approximation coefficients contain most of the energy of the signal. Hence, these coefficients are excluded in the threshold operation [26]. Let, $\mathrm{C}_{\mathrm{ECG}}[\mathrm{n}]$ is the noisy PRC and $\mathrm{C}_{\mathrm{ECG}}[\mathrm{n}]$ denotes the wavelet de-noised IMF then,

$$
\bar{C}_{E C G}[n]=\operatorname{Thr}\left[C_{E C G}[n], \varepsilon\right]
$$

Where Thr defines the thresholding operator and $\varepsilon$ is the threshold value. In this work, each IMF is decomposed into two levels by employing Symlet wavelet of order 7 (Sym7). Symlet family wavelets are much popular for signal de-noising because of their energy concentration at low frequency [27]. The threshold value is calculated according to Eq. (6), which is adopted from [28].

$$
\varepsilon=\sigma \sqrt{2 \log N}
$$

Where, $\sigma$ and $\mathrm{N}$ denote the standard deviation and the length ofthe detail wavelet coefficients at a particular level. Soft thresh-olding technique is employed in this work. The expression of soft thresholding is presented below

$$
\overline{\mathrm{d} w_{1}[1]}= \begin{cases}\left.\mid \mathrm{dw}_{\mathrm{i}}[1]\right]-\varepsilon_{1} & \left|\mathrm{dw}_{\mathrm{i}}[1]\right| \geq \varepsilon_{l} \\ 0 & \left|\mathrm{dw}_{\mathrm{i}}[1]\right| \leq \varepsilon_{l}\end{cases}
$$

Where, $\overline{d w_{1}[1]}$ and $d w_{i}[1]$ are the original and thresholded detail coefficients of level 1 .

\section{Signal reconstruction}

In this stage, the processed signal is restored by the adding the de-noised IMFs with the remaining IMFs and the residue signal.

$$
\begin{aligned}
& X_{E C G}[n]=\sum_{l=1}^{3} \overline{C_{l_{E C G}}}[n]+\sum_{l=4}^{L-1} C_{l_{E C G}}[n]+ \\
& r_{L}[n]
\end{aligned}
$$

\section{R-peak position information}

The locations of R-peaks in the ECG signal are extracted in this stage. This information will be helpful for correction of peaks after ASMF operation. Here, by employing any standard R-peak detection algorithm, the positions of R-peaks are extracted. In this work, standard Pan-Tompkins method [29] for QRS detection is utilized. This R-peak detection technique is well accepted and efficient for real-time applications.

\section{E. Adaptive switching mean filter (ASMF)}

After ITD based de-noising, some noises still exist in the reconstructed signal. These noises are clearly visible in the low time varying components of the ECG signal i.e. the region between QRS complexes. Hence, an adaptive switching mean filtering (ASMF) approach is applied for further enhancement of signal quality. ASMF is an efficient image filtering tool which is employed for removing the impulse noises from images [30]. The basic principle of ASMF is that there should be a similarity in the neighbourhood samples of a signal. In this method, a particular length of window is taken, and at each iteration, the centre of the window is placed on a test ECG sample. Now, a threshold value is estimated by calculating the standard deviation of the windowed region. If the difference between test ECG sample and the mean value of the windowed area is beyond the threshold limit, then it is considered as a corrupted sample, and its value is updated according to the mean value. The mathematical expression of the ASMF operation is demonstrated below.

$\bar{X}_{u}=\left\{\begin{array}{cr}m_{i} & \text { if }\left|X_{i}^{e}-m_{i}\right| \geq \alpha * \sigma_{i} \\ X_{i}^{e} & \text { else }\end{array}\right.$

Where $X_{i}^{e}$ and $\bar{X}_{i}$ are the input and processed ECG samples of ASMF operation, $m_{\bar{i}}$ and $\sigma_{\bar{i}}$ are the mean and standard deviation of thewindowed region. Here, $\alpha$ is a threshold selecting parameter, whichdetermines the limit of the threshold value. The value of $\alpha$ variesin between $0-1$. In this work, the value $\sigma_{i}$ is empirically taken 0.1 and a window of length 9 samples is chosen. The improvement of the signal quality after ASMF operation can be visualized.

\section{F. Peak correction.}

The R-peaks in the ECG signals are attenuated due to ASMF operation. These peaks carry vital medical information. Hence, there correction of these peaks is much essential. In this step, by utilizing the position information of R-peaks, the peaks are corrected according to the following algorithm.

\section{RESULTS AND DISSCUSSION}

The efficacy of the proposed work is evaluated using standard MIT-BIH arrhythmia database. This database consists a total 48 ECG records of duration 30 min each. Like the existing literature, in this work, the ECG records named as $100 \mathrm{~m}, 102 \mathrm{~m}, 103 \mathrm{~m}, 105 \mathrm{~m}, 200 \mathrm{~m}, 215 \mathrm{~m}$ have been used for simulation purpose. These signals contain time-varying QRS morphology, both normal and abnormal ECG beats. Electromyogram (EMG) noise at different SNR levels $(0 \mathrm{~dB}$, $5 \mathrm{~dB}, 10 \mathrm{~dB}, 15 \mathrm{~dB}$, and $20 \mathrm{~dB}$ ) is added to the signals. EMG noise is simulated by generating random noises as presented in [31].

\section{A. Qualitative Analysis}

In this subsection, a qualitative performance analysis of the proposed technique through visual inspection is carried out. Figure 3 shows the 100m ECG signal taken and signal after adding EMG noise at SNR level 10dB. We can observe that mostly low frequency components of the signal are affected due to noise.

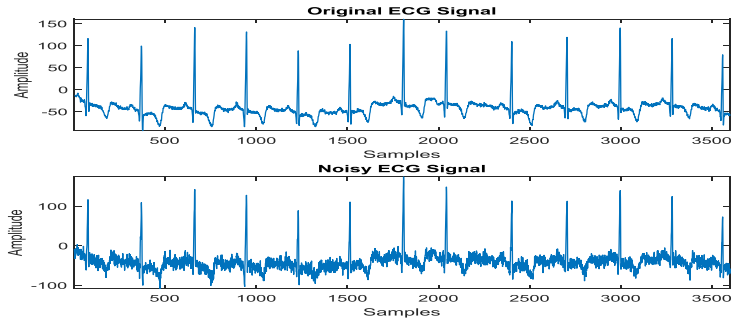

\section{Figure. 3. Original ECG signal and noise added ECG} signal

Figure .4 presents the de-noised ECG records 100m, which is corrupted by electromyogram noise at SNR level $10 \mathrm{~dB}$.

The de-noising proficiency of the proposed technique for an ECG signal which is corrupted with EMG noise is presented in Figure.5.

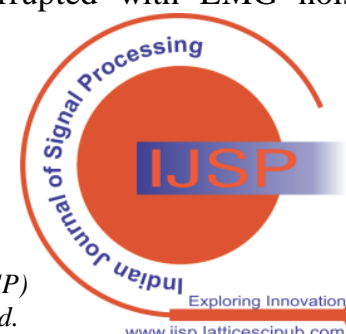


Here, ECG record $103 \mathrm{~m}$ is contaminated by EMG noise at SNR level $5 \mathrm{~dB}$. The figure illustrates that the technique efficiently rejects EMG noise from the signal and preserves the details.

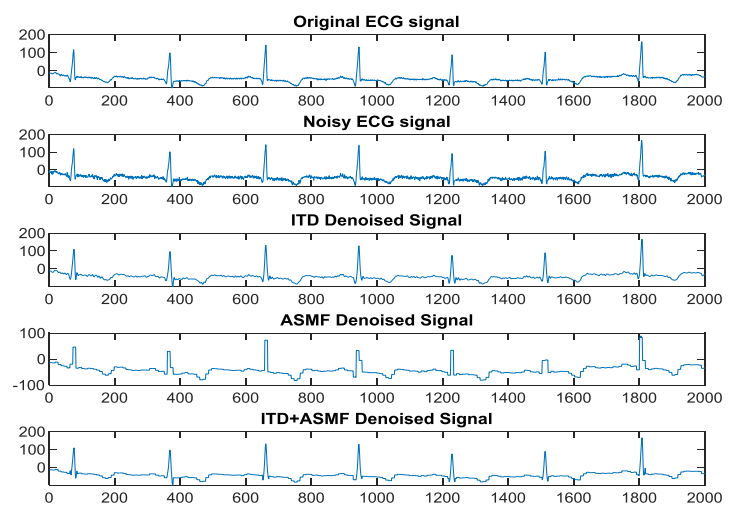

Figure. 4. De-noised ECG signal 100 m corrupted with electromyogram noise at input SNR level $10 \mathrm{~dB}$
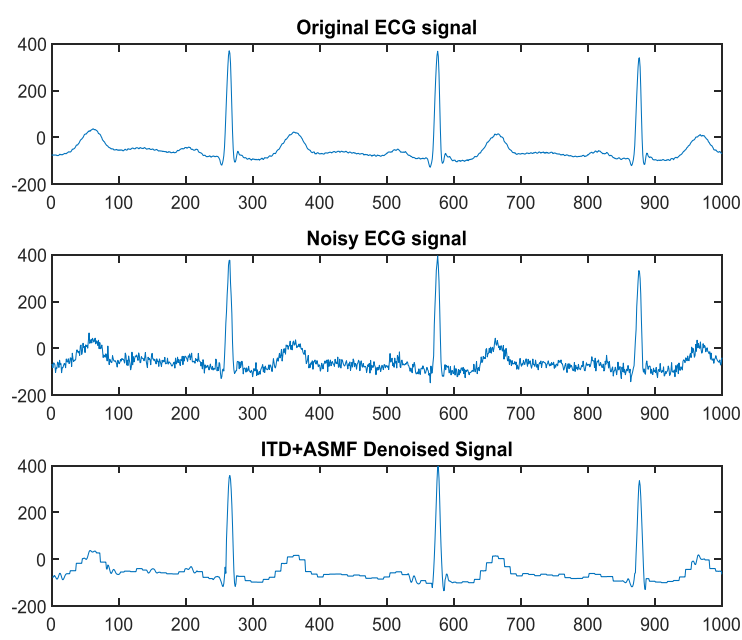

Figure. 5. De-noising of EMG noise corrupted 103m ECG signal at input SNR level 5dB
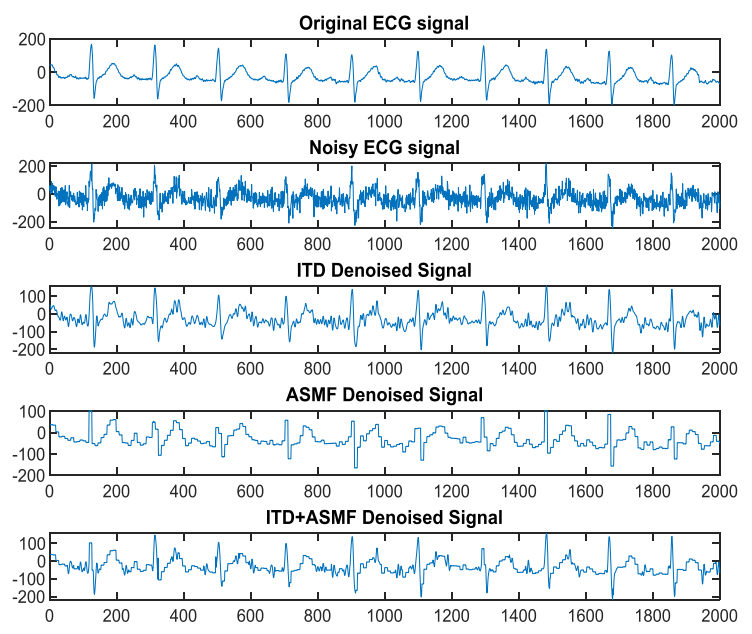

Figure. 6. De-noising of EMG noise corrupted 215m ECG signal at input SNR level 10dB

ECG record $215 \mathrm{~m}$ is contaminated by the EMG at SNR level $10 \mathrm{~dB}$. Figure 6 presents a qualitative analysis of the estimated signals through various methodologies. A visual inspection of the figure reveals that the proposed ASMF based technique can efficiently remove the effects of electromyogram and maintains all the characteristic details of the ECG signals.

\section{B. Quantitative analysis}

A quantitative performance analysis of the described technique is presented in this section. The performance metric namely output SNR improvement (SNRimp) is considered for evaluation. Signal to noise ratio (SNR) is the standard metric to quantify the quality of a signal from the energy perspective. SNR defines the signal energy with respect to the energy of the associated noise. SNRimp denotes the improvement of SNR value of a signal by the filtering process.

As the objective of this study to denoise the ECG signals, SNR improvement is a relevant performance metric to quantify the efficacy of the filter to reduce the effect of background noise. The performance parameter can be expressed as follows

$$
S N R_{\text {imp }}[d B]=10 \log _{10} \frac{\sum_{n=1}^{N}\left(X_{E C G}[n]-Y_{E C G}[n]^{*}\right.}{\sum_{n=1}^{N}\left(x_{E C G}^{f}[n]-Y_{E C G}[n]_{a}^{c}\right.}(10)
$$

Here, $Y_{E C G}[\mathrm{n}]$ denotes the original ECG, $X_{E C G}[\mathrm{n}]$ defines the corrupted signal, $X_{E C G}^{v}[\mathrm{n}]$ signifies the denoised ECG signal, and $\mathrm{N}$ is the length of the ECG signal.

Figure. 7 represents the output SNRimp of the denoised ECG signals considering electromyogram noise at input SNR $0 \mathrm{~dB}$ to $10 \mathrm{~dB}$ to $102 \mathrm{~m}$ ECG signal. The figure demonstrates that at each input SNR level the SNRimp of the proposed algorithm is higher than the other methods. The improvement of SNR in case of EMG noise corrupted ECG signals has been presented in Table-I and Table-II. It illustrates that the ASMF based methodology attains a better SNRimp than other techniques. Figure. 8 represents the output SNRimp of the denoised ECG signals considering electromyogram noise at input SNR $0 \mathrm{~dB}$ to $15 \mathrm{~dB}$ to $100 \mathrm{~m}$ ECG signal.

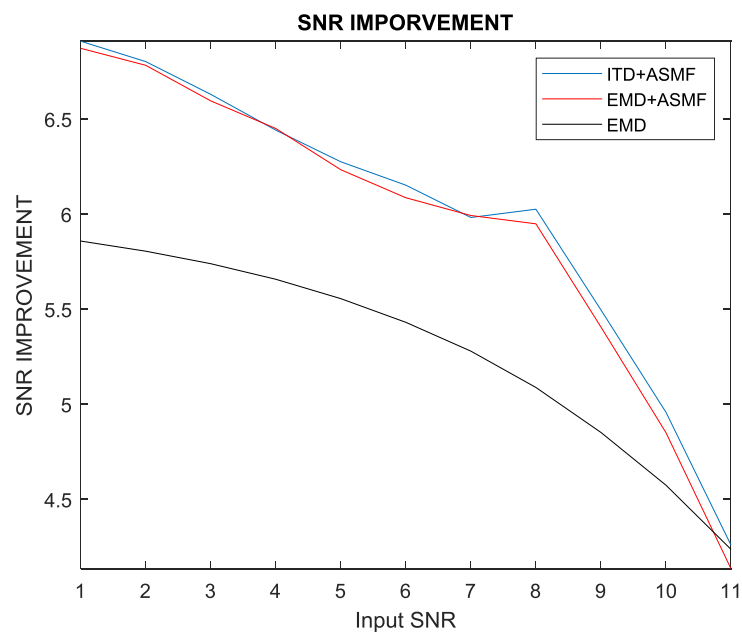

Figure. 7. SNR improvement results of the filtered $102 \mathrm{~m}$ signals for EMG noise

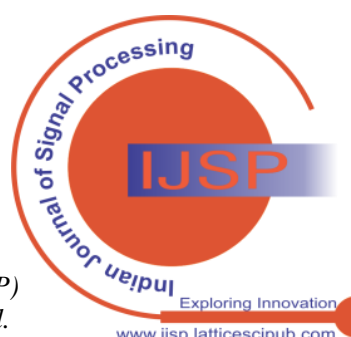


Table-I: SNR improvement for denoised $102 \mathrm{~m}$ signal

\begin{tabular}{|c|c|c|c|}
\hline $\begin{array}{c}\text { Technique } \rightarrow \\
\text { Input } \\
\text { SNR(dB) }\end{array}$ & $\begin{array}{c}\text { Proposed } \\
\text { Method }\end{array}$ & EMD+ASMF & EMD \\
\hline $\mathbf{0}$ & 6.909411 & 6.872059 & 5.858497 \\
\hline $\mathbf{1}$ & 6.802157 & 6.782630 & 5.805119 \\
\hline $\mathbf{2}$ & 6.629933 & 6.595575 & 5.739361 \\
\hline $\mathbf{3}$ & 6.441835 & 6.450309 & 5.657563 \\
\hline $\mathbf{4}$ & 6.275409 & 6.233482 & 5.555631 \\
\hline $\mathbf{5}$ & 6.152309 & 6.086323 & 5.431142 \\
\hline $\mathbf{6}$ & 5.982487 & 5.992816 & 5.279565 \\
\hline $\mathbf{7}$ & 6.025934 & 5.949103 & 5.088632 \\
\hline $\mathbf{8}$ & 5.496466 & 5.406565 & 4.852005 \\
\hline $\mathbf{9}$ & 4.958191 & 4.851009 & 4.574653 \\
\hline $\mathbf{1 0}$ & 4.259635 & 4.135417 & 4.237286 \\
\hline
\end{tabular}

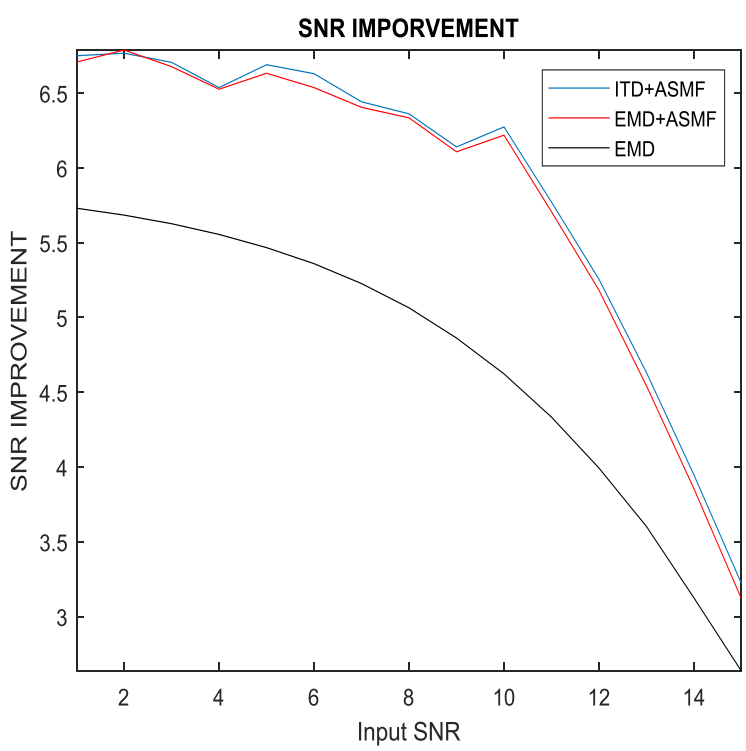

Figure. 8. SNR Improvement for denoised $100 \mathrm{~m}$ signal

Table-II: SNR improvement for denoised $100 \mathrm{~m}$ signal

\begin{tabular}{|c|c|c|c|}
\hline Technique $\rightarrow$ & $\begin{array}{c}\text { Proposed } \\
\text { Method }\end{array}$ & EMD+ASMF & EMD \\
\hline $\begin{array}{c}\text { Input } \\
\text { SNR(dB) } \downarrow\end{array}$ & & & \\
\hline $\mathbf{0}$ & 6.749807 & 6.707672 & 5.730525 \\
\hline $\mathbf{1}$ & 6.766868 & 6.788194 & 5.684515 \\
\hline $\mathbf{2}$ & 6.705868 & 6.676929 & 5.626899 \\
\hline $\mathbf{3}$ & 6.536951 & 6.526095 & 5.555298 \\
\hline $\mathbf{4}$ & 6.690074 & 6.632716 & 5.466927 \\
\hline $\mathbf{5}$ & 6.629914 & 6.536899 & 5.359743 \\
\hline $\mathbf{6}$ & 6.442063 & 6.405118 & 5.226751 \\
\hline $\mathbf{7}$ & 6.361498 & 6.334518 & 5.064465 \\
\hline $\mathbf{8}$ & 6.140281 & 6.107708 & 4.862674 \\
\hline $\mathbf{9}$ & 6.274098 & 6.218774 & 4.623086 \\
\hline $\mathbf{1 0}$ & 5.771227 & 5.707140 & 4.333545 \\
\hline
\end{tabular}

\section{CONCLUSION}

In this project, an efficient combined ECG de-noising methodology using ITD and ASMF is proposed. The main aim of this work is to incorporate the advantages of both ITD and ASMF in reducing the noises present in ECG signals. In general ECG de-noising techniques, reject initial PRCs or window-based approach is employed for reduction of high-frequency noises. However, these approaches either harm the high-frequency details of ECG signals or ignore the noises in QRS complex region. Unlike, conventional methods, here, a wavelet based soft thresholding technique is applied for rejection of high-frequency noises and preservation of QRS complexes. Then, an ASMF operation is employed for enhancing the signal quality by further removing of artifacts. So combining these two scheme yield better ECG de-noising.In the proposed approach the ECG signal is decomposed into a sum of components with slowly varying amplitudes and frequencies using the ITD. It can be seen from the simulation results that the proposed technique performs better than the EMD-based approach for EMG noise removal.

\section{REFERENCES}

1. S. Shadmand, B. Mashoufi, A new personalized ECG signal classificationalgorithm using block-based neural network and particle swarmoptimization, Biomed. Signal Process. Control 25 (2016) 12-23, http://dx.doi.org/10.1016/j.bspc.2015.10.008. [CrossRef]

2. S. Farashi, A multiresolution time-dependent entropy method for QRScomplex detection, Biomed. Signal Process. Control 24 (2016) 63-71, http://dx.doi.org/10.1016/j.bspc.2015.09.008. [CrossRef]

3. R. Tung, P. Zimetbaum, Use of the Electrocardiogram in Acute MyocardialInfarction, Card. Intens. Care (2010) 106-109, http://dx.doi.org/10.1016/B978-1-4160-3773-6.10011-4. [CrossRef]

4. K. Sailunaz, M. Alhussein, M. Shahiduzzaman, F. Anowar, K.A. Al Mamun,CMED Cloud based medical system framework for rural health monitoring indeveloping countries, Comput. Electr. Eng. 53 (2016) 469-481, http://dx.doi.org/10.1016/j.compeleceng.2016.02.005. [CrossRef]

5. M.Z.U. Rahman, R.A. Shaik, D.V. Rama Koti Reddy, Efficient sign basednormalized adaptive filtering techniques for cancelation of artifacts in ECGsignals: application to wireless biotelemetry, Signal Processing http://dx.doi.org/10.1016/j.sigpro.2010.07.002. [CrossRef]

6. S. Pal, M. Mitra, Empirical mode decomposition based ECG enhancement andQRS detection, Comput. Biol. Med. 42 (2012) 83-92, [CrossRef

7. V.X. Afonso, W.J. Tompkins, T.Q. Nguyen, K. Michler, S. Luo, Comparing stressECG enhancement algorithms, Eng. Med. Biol. Mag IEEE 15 (1996) 37-44,http://dx.doi.org/10.1109/51.499756. [CrossRef]

8. I.I. Christov, I.K. Daskalov, Filtering of electromyogram artifacts from theelectrocardiogram, Med. Eng. Phys. 21 (1999) 731-736, http://dx.doi.org/10.1016/S1350-4533(99)00098-3. [CrossRef]

9. S. Poungponsri, X.-H. Yu, An adaptive filtering approach for electrocardiogram(ECG) signal noise reduction using neural networks, Neurocomputing 117(2013) 206-213, http://dx.doi.org/10.1016/j.neucom.2013.02.010. [CrossRef]

10.E. Gokgoz, A. Subasi, Effect of multiscale PCA de-noising on EMG signalclassification for diagnosis of neuromuscular disorders, J. Med. Syst. $\quad 38(2014), \quad$ http://dx.doi.org/10.1007/s10916-014-0031-3. [CrossRef]

11.T. He, G. Clifford, L. Tarassenko, Application of independent componentanalysis in removing artefacts from the electrocardiogram, Neural Comput.Appl. $15 \quad$ (2006) 105-116 http://dx.doi.org/10.1007/s00521-005-0013-y. [CrossRef]

12.B.H. Tracey, E.L. Miller, Nonlocal means denoising of ECG signals, IEEE Trans.Biomed. Eng. 59 (2012) 2383-2386, http://dx.doi.org/10.1109/TBME.2012.2208964. [CrossRef]

13. R. Sameni, M.B. Shamsollahi, C. Jutten, G.D. Clifford, A nonlinear bayesianfiltering framework for ECG denoising, IEEE Trans. Biomed. Eng. $54 \quad$ (2007)2172-2185, http://dx.doi.org/10.1109/TBME.2007.897817. [CrossRef]

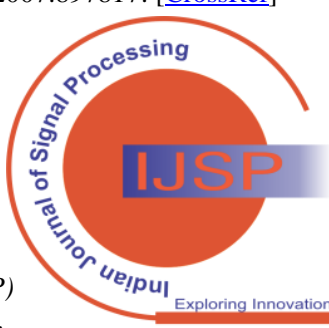


14. E. Erc, elebi, Electrocardiogram signals de-noising using lifting-based discretewavelet transform, Comput. Biol. Med. 34 (2004) 479-493, http://dx.doi.org/10.1016/S0010-4825(03)00090-8. [CrossRef]

15.S. Poornachandra, Wavelet-based denoising using subband dependentthreshold for ECG signals, Digital Signal Process. 18 (2008) 49-55, http://dx.doi.org/10.1016/j.dsp.2007.09.006. [CrossRef]

16. B.N. Singh, A.K. Tiwari, Optimal selection of wavelet basis function applied toECG signal denoising, Digital Signal Process. 16 (2006) 275-287, http://dx.doi.org/10.1016/j.dsp.2005.12.003. [CrossRef]

17. M. Blanco-Velasco, B. Weng, K.E. Barner, ECG signal denoising and baselinewander correction based on the empirical mode decomposition, Comput. Biol.Med. 38 (2008) 1-13, http://dx.doi.org/10.1016/j.compbiomed.2007.06.003. [CrossRef]

18. M.A. Kabir, C. Shahnaz, Denoising of ECG signals based on noise reductionalgorithms in EMD and wavelet domains, Biomed. Signal $\begin{array}{llll}\text { Process. } & \text { Control 4(2012) 481-489, }\end{array}$ http://dx.doi.org/10.1016/j.bspc.2011.11.003. [CrossRef]

19. W. Jenkal, R. Latif, A. Toumanari, A. Dliou, O. El B'Charri, F.M.R. Maoulainine,An efficient algorithm of ECG signal denoising using the adaptive dualthreshold filter and the discrete wavelet transform, Biocybern. Biomed. Eng.36 (2016) 499-508, http://dx.doi.org/10.1016/j.bbe.2016.04.001. [CrossRef]

20.H. Liang, Q.H. Lin, J.D.Z. Chen, Application of the empirical modedecomposition to the analysis of esophageal manometric data ingastroesophageal reflux disease, IEEE Trans. Biomed. Eng. 52 (2005)1692-1701， http://dx.doi.org/10.1109/TBME.2005.855719. [CrossRef]

21.G.B. Moody, R.G. Mark, The impact of the MIT-BIH arrhythmia database, IEEEEng. Med. Biol. Mag. 20 (2001) 45-50, http://dx.doi.org/10.1109/51.932724. [CrossRef]

22. A. Dasgupta, S. Chakraborty, A. Routray, A two-stage framework for denoisingelectrooculography signals, Biomed. Signal Process. Control 31 (2017)231-237, http://dx.doi.org/10.1016/j.bspc.2016.08.012. [CrossRef]

23. N. Huang, Z. Shen, S. Long, M. Wu, H. SHIH, Q. ZHENG, N. Yen, C. Tung, H. Liu,The empirical mode decomposition and the Hilbert spectrum for nonlinearand non-stationary time series analysis, Proceedings of Royal society AMathematics Physical and Engineering $\begin{array}{llll}\text { Sciences } & 454 & \text { (1998) } & 903-995\end{array}$ http://dx.doi.org/10.1098/rspa.1998.0193. [CrossRef]

24. S. Liu, L. Xu, D. Li, Multi-scale prediction of water temperature using empiricalmode decomposition with back-propagation neural networks, Comput. $\quad$ Electr.Eng. $49 \quad$ (2016) 1-8, http://dx.doi.org/10.1016/j.compeleceng.2015.10.003. [CrossRef]

25. T. Wang, L. Lin, A. Zhang, X. Peng, C.A. Zhan, EMD-based EEG signalenhancement for auditory evoked potential recovery under highstimulus-rate paradigm, Biomed. Signal Process. Control 8 (2013) 858-868,http://dx.doi.org/10.1016/j.bspc.2013.08.004. [CrossRef]

26. M. Alfaouri, K. Daqrouq, ECG signal denoising by wavelet transformthresholding, Am. J. Appl. Sci. 5 (2008) 276-281, http://dx.doi.org/10.3844/ajassp.2008.276.281. [CrossRef]

27. S. Mahmoodabadi, a Ahmadian, M. Abolhasani, M. Eslami, J. Bidgoli, ECGfeature extraction based on multiresolution wavelet transform, Conference Proceedings of IEEE Engineering in Medicine Biology Society 4 (2005)3902-3905, http://dx.doi.org/10.1109/IEMBS.2005.1615314. [CrossRef]

28.D.L. Donoho, De-noising by soft-thresholding, IEEE Trans. Inf Theory 41(1995) 613-627, http://dx.doi.org/10.1109/18.382009.

29. J. Pan, W.J. Tompkins, A real-time QRS detection algorithm, IEEE Trans.Biomed. $\quad$ Eng. $32 \quad$ (1985) 230-236, http://dx.doi.org/10.1109/TBME.1985.325532. [CrossRef]

30. V. Gupta, V. Chaurasia, M. Shandilya, Random-valued impulse noise removalusing adaptive dual threshold median filter, J. Vis. Commun. Image Represent.26 (2015) 296-304, http://dx.doi.org/10.1016/j.jvcir.2014.10.004. [CrossRef]

31.J. Wang, Y. Ye, X. Pan, X. Gao, Parallel-type fractional zero-phase filtering forECG signal denoising, Biomed. Signal Process. Control 18 (2015) 36-41, http://dx.doi.org/10.1016/j.bspc.2014.10.012. [CrossRef]

32.H. Mamaghanian, N. Khaled, D. Atienza, P. Vandergheynst, Compressedsensing for real-time energy-efficient ECG compression on wireless bodysensor nodes, IEEE Trans. Biomed. Eng. 58 (2011) 2456-2466, http://dx.doi.org/10.1109/TBME.2011.2156795. [CrossRef]

33. M.S. Manikandan, K.P. Soman, A novel method for detecting R-peaks inelectrocardiogram (ECG) signal, Biomed. Signal Process. Control 7 (2012)118-128, http://dx.doi.org/10.1016/j.bspc.2011.03.004. [CrossRef]

Feldman, M.: 'Time-varying vibration decomposition and analysis based on the Hilbert transform', J. Sound Vib., 2006, 295, 3-5, pp. 518-530. [CrossRef]

\section{AUTHORS PROFILE}

Dr Battula Tirumala Krishna professor of Electronics and Communication Engineering Department JNTUK Kakinada Tkbattula@gmail.com.

Putti Siva Kameswaari M.Tech from Electronics and Communication Engineering Department JNTUK Kakinada siva9putti@gmail.com

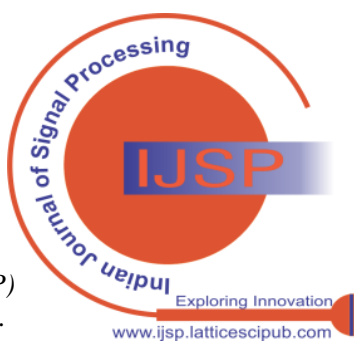

